DOT/FAA/AM-08/10

Office of Aerospace Medicine

Washington, DC 20591

\title{
Drug Usage in Pilots Involved in Aviation Accidents Compared With Drug Usage in the General Population: From 1990 to 2005
}

Sabra R. Botch

Robert D. Johnson

Civil Aerospace Medical Institute Oklahoma City, OK 73125

April 2008

Final Report 


\section{NOTICE}

This document is disseminated under the sponsorship of the U.S. Department of Transportation in the interest of information exchange. The United States Government assumes no liability for the contents thereof.

This publication and all Office of Aerospace Medicine technical reports are available in full-text from the Civil Aerospace Medical Institute's publications Web site: www.faa.gov/library/reports/medical/oamtechreports/index.cfm 
Technical Report Documentation Page

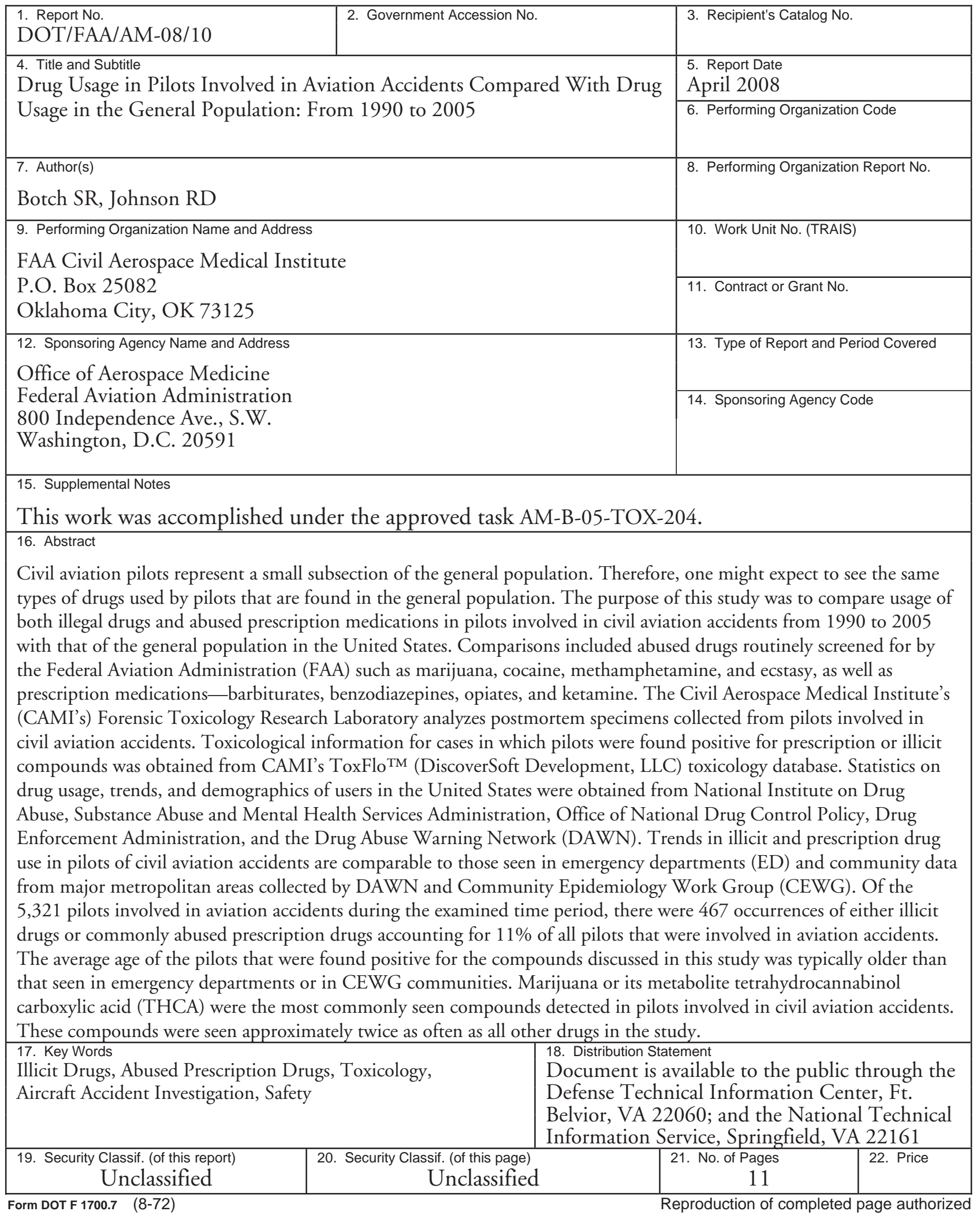





\section{Drug Usage in Pilots Involved in Aviation Accidents Compared With Drug Usage in the General Population: From 1990 to 2005}

\section{INTRODUCTION}

Civil aviation pilots represent a small subsection of the general population. Therefore, one might expect to see the same types of drugs used by pilots that are found in the general population. The purpose of this study was to compare usage of both illegal drugs and abused prescription medications in pilots involved in civil aviation accidents from 1990 to 2005 with that of the general population in the United States. Comparisons included abused drugs routinely analyzed for by the Federal Aviation Administration (FAA) such as marijuana, cocaine, methamphetamine, and ecstasy, as well as prescription medications - barbiturates, benzodiazepines, opiates, and ketamine. Each year was evaluated for pilots that were found positive for the previously mentioned drugs. Additional factors, as available, such as usage per year, age, gender, and concomitant use of multiple substances were compared with statistics on drug usage for individuals in the United States.

\section{METHODS}

The Civil Aerospace Medical Institute's (CAMI's) Forensic Toxicology Research Laboratory analyzes postmortem specimens collected from pilots involved in civil aviation accidents. ${ }^{1,2}$ Toxicological information for cases in which pilots were found positive for prescription or illicit compounds was obtained from CAMI's ToxFlo ${ }^{\mathrm{TM}}$ (DiscoverSoft Development, LLC, Oklahoma City, OK) toxicology database. Statistics on drug usage, trends, and demographics of users in the United States were obtained from the National Institute on Drug Abuse (NIDA), Substance Abuse and Mental Health Services Administration (SAMHSA), Office of National Drug Control Policy (ONDCP), Drug Enforcement Administration (DEA), Drug Abuse Warning Network (DAWN), Community Epidemiology Work Group (CEWG), and the National Survey on Drug Use and Health (NSDUH).

For drug classification purposes, the schedule in which a compound is listed in the Controlled Substances Act (CSA) was included in this study. ${ }^{3}$ The CSA places all substances that are in some manner regulated under existing federal law into one of five schedules. This placement is based upon the substance's medical use, potential for abuse, and safety or dependence liability.
The determination into which schedule a drug or other substance should be placed, decontrolled, or rescheduled is based upon consideration of several factors. These factors include the potential for abuse, scientific evidence of the drug's pharmacological effect(s), pattern of abuse, significance/scope of abuse, risk to public health, psychological and physiological dependence liability, and whether the substance is an immediate precursor of another substance that is already controlled. Drugs placed in Schedule I have a high potential for abuse and no medical purpose. Those in Schedule II have a medical purpose and a high abuse potential. Schedule III drugs have a medical use and have less potential for abuse relative to those in Schedules I or II. Schedule IV drugs have a low potential for abuse relative to Schedule III and a medical use. Drugs in Schedule V have a low potential for abuse relative to drugs in Schedule IV and a currently accepted medical use.

\section{EXAMINED SUBSTANCES}

\section{Marijuana}

Marijuana is the most commonly abused illicit drug in the United States and is listed as a Schedule I substance under the CSA., ${ }^{3,4}$ Marijuana is obtained from various parts of the Cannabis sativa plant. It can be seen in numerous forms, including hashish, hash oil, bhang, and ganja. The active chemical compound in marijuana is $\Delta$-9-tetrahydrocannabinol (THC). THC and/or tetrahydrocannabinol carboxylic acid (THCA) (its major metabolite) was detected in 122 male pilots over the examined time period. It was detected on average in 8 pilots per year. In the years 1994, 1996, and 2003 it was detected in more than 10 pilots each year, with an average age of 40 years (range 18 to 71 ).

In 1994, SAMHSA conducted a National Household Survey and found that in an average month in 1994, 10 million Americans used marijuana, making it the most commonly used illicit drug. The average age of current illicit drug users and the proportion of them that are age 35 and older have risen steadily since 1979. The occurrence of THC and/or THCA in pilots was relatively stable over the years examined. As with the general population, THC was the most commonly detected illicit drug in pilots involved in civil aviation accidents. 


\section{Cocaine}

Cocaine, listed as a Schedule II drug in the CSA, has a high potential for abuse but may be administered for legitimate medical reasons, such as a local anesthesia for some eye, ear, and throat surgeries. ${ }^{3}$ With the exception of 2002, cocaine or its major metabolite benzoylecgonine was detected in pilots at least once each year. Cocaine was detected in 41 male pilots over the examined time period and was seen in 6 male pilots in both 1994 and 2001. The average age of the pilots was 41 years (range 27 to 57 ).

In 1994, according to the national Household Survey on Drug Abuse, an estimated 1.4 million Americans were using cocaine; that is, they had used cocaine at least once in the past month. ${ }^{5}$ Of that number, an estimated onehalf million were current crack users. ${ }^{5}$ The rate of current cocaine use was highest among young adults. In 1994, approximately $60 \%$ of current users were ages 18 through 34. ${ }^{5}$ Data from DAWN, which collects information on drug-related episodes from hospital emergency departments in 21 metropolitan areas, showed that cocainerelated emergency department $(E D)$ visits increased 33\% between 1995 and 2002, rising from 58 to 78 mentions per 100,000 population. ${ }^{6}$ Cocaine was the second-most commonly detected illicit drug in male pilots, which is congruent with that seen in ED mentions from data collected from DAWN.?

\section{Methamphetamine}

Methamphetamine is a Schedule II stimulant with a high potential for abuse. This compound has limited medical uses, but it may be used to treat narcolepsy, attention deficit disorders, and obesity. ${ }^{3}$ Methamphetamine was detected at least once each year in a male pilot from 1991 - 2002, 2004, and 2005. The average age of the pilots was 43 years (Range $25-78$ ). Data from DAWN from the year 2000 reported that methamphetaminerelated episodes increased from approximately 10,400 in 1999 to 13,500 in 2000, a 30\% increase. ${ }^{7}$ The years 2000 and 2001 had the greatest number of pilots that tested positive for methamphetamine (3 each year). This trend follows that of the general population. Based on data from the 2001 NSDUH, there was no significant increase in methamphetamine usage in the 40 to 44 year age category during this period (2000 to 2001). ${ }^{8}$

\section{MDMA (Ecstasy)}

MDMA (3,4-methylenedioxymethamphetamine) is a synthetic, psychoactive drug chemically similar to the stimulant methamphetamine and the hallucinogen mescaline. MDMA is listed as a Schedule I drug in the CSA. According to the $2005 \mathrm{NSDUH}$, more than 11 million people have tried this drug at least once. ${ }^{9}$ MDMA first gained popularity among adolescents and young adults in the nightclub scene or weekend-long dance parties known as raves. However, community-level data from CEWG continued to report that the use of MDMA had spread among populations outside the nightclub scene. ${ }^{9}$ MDMA was detected once during the examined time period in a pilot fatality. This occurred in the year 2000. The 27-year-old male student pilot was also found positive for benzoylecgonine (active metabolite of cocaine), methamphetamine, and amphetamine. Based on data compiled by DAWN, MDMA-related emergency department visits increased significantly from 2,850 in 1999 to 4,511 in 2000 . The majority of such visits for MDMA use were from individuals under the age of 26 years (75\%)..$^{10}$ MDMA use by victims of aviation accidents was rare. The occurrence in 2000 accounted for $<1 \%$ of the total fatal aviation accidents for that year (323 accidents) and $<0.01 \%$ during the examined time period (5192 accidents).

\section{Benzodiazepines}

Psychotherapeutic agents, anxiolytics, sedatives, and hypnotics occurred in 34\% of emergency department visits associated with nonmedical use of pharmaceuticals. ${ }^{7}$ Emergency department (ED) visits involving benzodiazepines outnumber those involving any of the other types of psychotherapeutic agents. ${ }^{7}$ DAWN estimated that in 2005 there were $172,388 \mathrm{ED}$ visits associated with nonmedical use of benzoydiazepines. ${ }^{11}$ ED visit rates for nonmedical use of pharmaceuticals did not differ between females and males, with the highest rates for patients aged 18 to $54 .{ }^{11}$ Visits related to nonmedical use of pharmaceuticals increased $21 \%$ from 2004 to $2005 .{ }^{11}$ ED mentions of benzodiazepines increased 14\% (from 91,078 to 103,972) from 2000 to 2001 . $^{7}$ From 1994 to 2001, mentions of benzodiazepines in ED visits escalated by $39 \% .^{7}$ In the pilot community, benzodiazepine use has remained steady, with a slight increase in 1996, followed by a return to the rate seen in previous years.

\section{Diazepam}

Diazepam, commonly prescribed as Valium ${ }^{\circledR}$, is used to relieve anxiety, muscle spasms, and seizures and to control agitation caused by alcohol withdrawal. A Schedule IV benzodiazepine, it works by enhancing the effects of gamma amino butyric acid (GABA). ${ }^{3}$ Diazepam was detected in at least one male pilot every year, with the exception of 2003, in which it was not detected. In 1996, diazepam was detected in 6 male pilots; the average number of pilots per year that tested positive for diazepam was 2 . The average age of the pilots using diazepam was 49 years (range 17-74). Of the 34 male pilots that tested positive for diazepam, $8(-24 \%)$ were age 65 or older. In 
the U. S. those 65 years of age and above comprise only $13 \%$ of the population, yet account for approximately $33 \%$ of all medications prescribed. ${ }^{13}$

\section{Alprazolam}

Alprazolam $\left(\operatorname{Xanax}^{\circledR}\right)$, a benzodiazepine, is used to treat anxiety disorders and panic attacks. It works by decreasing abnormal excitement in the brain. The CSA lists alprazolam as a schedule IV drug. ${ }^{3}$ Alprazolam or its major metabolite alpha-hydroxyalprazolam was seen at least once during 1993, 1994, 1997, 2000, 2001, and 2002 in male pilots. The average age of the pilots using alprazolam was 46 (range 30-70). Based on data evaluated by the CEWG, alprazolam was identified as the most frequently abused benzodiazepine in most areas of the $\mathrm{U}$. S. ${ }^{12}$ However, diazepam was the most common benzodiazepine detected in pilots and was detected 3 times more often than alprazolam (33-diazepam, 9-alprazolam).

\section{Barbiturates}

In 2001, the barbiturates most frequently reported to DAWN were barbiturates-not otherwise specified (NOS; 7,209 mentions). ${ }^{11}$ Barbiturate-NOS is a category used to document use of a class of compounds when the exact barbiturate is not specified. ${ }^{11}$ Mentions of barbituratesNOS increased 49\% from 2000 to 2001 and has increased $153 \%$ since $1994 .{ }^{11}$ Phenobarbital was the second most frequent barbiturate reported to DAWN in 2001 (1,117 mentions). ${ }^{11}$ Phenobarbital mentions have decreased $55 \%$ since 1994 . According to the 2003 NSDUH, an estimated 4.7 million Americans used prescription drugs non-medically for the first time in 2002: 2.5 million used pain relievers, 1.2 million used tranquilizers, 761,000 used stimulants, and 225,000 used sedatives. ${ }^{13}$ With the exception of 1991 and 2002, barbiturate occurrence in pilots was low and averaged 2 per year. In 1991 and 2002, barbiturates were detected in 5 or more pilots.

\section{Pentobarbital}

Pentobarbital, a Schedule II barbiturate, is used for the short-term treatment of insomnia. ${ }^{3}$ It is also used as a sedative to relieve anxiety and induce sleep before surgery. It belongs to a group of medicines called central nervous system (CNS) depressants that induce drowsiness and relieve tension or nervousness. Pentobarbital binds at a distinct binding site associated with a $\mathrm{Cl}^{-}$ionopore at the $\mathrm{GABA}_{\mathrm{A}}$ receptor. Pentobarbital was detected in 6 male pilots (1991-1, 1997-3, 2002-2). The average age of the pilots using pentobarbital was 52 years (range 37-68). In each of these pilots, at least one other prescription or over-the-counter (OTC) drug was detected in combination with pentobarbital.

\section{Phenobarbital}

Phenobarbital is used to control epilepsy and, as a sedative, to relieve anxiety. It is also used for short-term treatment of insomnia. Phenobarbital is a Schedule IV drug. ${ }^{3}$ It alters sensory cortex, cerebellar, and motor activities; produces sedation, hypnosis, and anesthesia. Phenobarbital was detected in 6 male pilots (1991-2, 1992-2, 1993-1, 1999-1) with an average age of 63 years (range 46-86).

\section{Butalbital}

Butalbital was seen in 17 male pilots: 1991-3, 19942, 1995-2, 1996-1, 1999-1, 2000-1, 2001-1, 2002-3, and 2005-3. The average age was 65 (range 32-69). In 8 out of the 17 pilots that tested positive for butalbital, acetaminophen or salicylate was also detected. The combination's of butalbital with acetaminohen (trade names: Axocet $^{\circledR}$, Bucet $^{\circledR}$, Bupap $^{\circledR}$, Cephadyn $^{\circledR}$ ) and butalbital with salicylate (trade name: $\operatorname{Axotal}^{\circledR}$ ) are used to treat tension headaches. Butalbital is listed as a Schedule III drug. ${ }^{3}$

\section{Secobarbital}

Secobarbital is used in the short-term treatment of insomnia and is a Schedule II drug. ${ }^{3}$ Secobarbital was seen only once and it was in a 51-year-old male pilot in 1994.

\section{Opiates}

A major finding from the CEWG 2000-2001 reporting period revealed that the use of opiates, other than heroin, continued to increase; specifically, the abuse of hydrocodone and oxycodone. ${ }^{14}$ During the same time period, the number of drug abuse related death mentions in DAWN for opiate analgesics peaked in 16 CEWG areas and exceeded those for cocaine and heroin in 8 areas. ${ }^{14}$

Data complied by DAWN for the coterminous United States in 2000 show that narcotic analgesics and narcotic analgesic combinations were the most frequently mentioned CNS agents in drug-related emergency department visits. ${ }^{14}$ Most often mentioned were narcotic analgesics containing hydrocodone (20,098 mentions), oxycodone $(10,825)$, methadone $(7,819)$, propoxyphene $(5,485)$, codeine $(5,295)$, and meperidine $(1,085) .{ }^{14}$ DAWN's review of data for 2000 and 2001 shows a $44 \%$ increase in the number of oxycodone mentions (from 5,437 to 7,831 ) and a $38 \%$ decrease in the number of codeine mentions (from 2,578 to 1,593). ${ }^{14}$

Long-term increases in the number of narcotic analgesics ED mentions were significant for hydrocodone (116\% from 1994 to 2000), oxycodone (166\%), and methadone (140\%). ${ }^{14}$ Based on the number of narcotic analgesic mentions in DAWN and CEWG areas between 1997 and 2000, over half of the 20 metropolitan areas 
saw an increase in opiod analgesic-related deaths from 1998 to $1999 .{ }^{14}$ Per NIDA, in 20034.7 million Americans reported using pain relievers such as codeine and other opiates for non-medical purposes. ${ }^{12}$ Pain reliever incidence increased-from 573,000 initiates in 1990 to 2.5 million initiates in 2000-and has remained stable through $2003 .{ }^{13}$

Opiates are the second-most commonly detected compounds in pilots. In a given year the average number of pilots testing positive for an opiate was 8. From 1998 to 1999 , a large increase was seen in opiates detected in pilots (5-1998, 12-1999). The number of pilots found positive for opiates remained constant and high from 1999 to 2003, when the average increased to 15 per year. This is comparable to the trend seen in the general population.

\section{Morphine}

Morphine, a Schedule II drug, is an opiate (narcotic) analgesic that is used to relieve moderate to severe pain. ${ }^{3}$ Morphine is used parenterally for preoperative sedation, as a supplement to anesthesia, and for analgesia. The detection of morphine in a male pilot has occurred at least once per year, with 9 occurrences in 2003, 7 in 2001, and 7 in 1999. The average number of pilots per year that tested positive for morphine was 4 . The average age of these pilots was 52 years (range 25 to 78 ).

\section{Hydrocodone/Hydromorphone}

Hydrocodone is an antitussive and analgesic agent for the treatment of moderate to severe pain. Hydrocodone is as effective for cough suppression and similar to morphine when used for pain relief. ${ }^{15}$ The DEA lists hydrocodone as the most frequently prescribed opiate in the United States with over 124 million prescriptions for hydrocodone-containing products dispensed in 2005. ${ }^{15}$ Hydromorphone is a Schedule II opioid analgesic and is a minor metabolite of hydrocodone. ${ }^{3}$ According to DAWN, the estimated number of emergency department episodes involving hydromorphone increased from 937 in 1996 to 2,667 in 2002..$^{15}$

Hydrocodone is often prescribed as Vicodin ${ }^{\circledR}$ or Lortab $^{\circledR}$, which is the combination of hydrocodone and acetaminophen. Of the 25 male pilots in which hydrocodone was detected, acetaminophen was also detected in 16. The average age of these pilots was 54 years (range 29 to 81 ). Hydromorphone was detected with hydrocodone in 14 of the pilots. In 2002, hydrocodone was detected in 6 male pilots. It was detected at least once each year, with the exception of 1991, 1992, 1994, 1998, 2002, and 2005.

\section{Codeine}

Codeine is medically prescribed for the relief of moderate pain and cough suppression. Compared to morphine, codeine produces less analgesia, sedation, and respiratory depression, and is usually taken orally. Depending on the type of preparation (i.e. alone as a tablet, in combination with aspirin/acetaminophen, or in liquid preparations) it can be listed as a Schedule II, III, or V drug. ${ }^{3}$ Codeine was detected in 14 male pilots with an average age of 53 years (range 26 to 67). It was detected at least once in 1991, 1992, 1995, 1996, 1998, 2000, 2001, 2002, 2003, and 2005. In 1999, it was detected in 3 male pilots; and in 2 male pilots in 2003 .

\section{Fentanyl}

Fentanyl is a powerful synthetic opiate analgesic similar to, but more potent than, morphine. It is typically used to treat patients with severe pain or to manage pain after surgery. It is also sometimes used to treat people with chronic pain who are physically tolerant to opiates. Fentanyl is a Schedule II prescription drug. ${ }^{3}$ Fentanyl was seen in two male pilots: one age 26 in 1996 and one age 44 in 2005.

\section{Other Narcotic Analgesics}

Meperidine and propoxyphene were rarely detected in pilots involved in aviation accidents. Meperidine and dextropropoxyphene (propoxyphene in non-dosage form) are considered Schedule II drugs. ${ }^{3}$ Meperidine $\left(\right.$ Demerol $\left.^{\circledR}\right)$ is a rapid-acting opioid analgesic. Propoxyphene $\left(\right.$ Darvon $\left.^{\circledR}\right)$ is a mild analgesic, structurally as well as pharmacologically similar to methadone; in dosage form is considered a Schedule IV drug. ${ }^{3}$

During the examined time period, meperidine was detected in one male pilot in the years 1994, 1995, 2000 and 2002. The average age was 53 (range $40-70$ ). Propoxyphene was detected in at least one male pilot each year from 1995 to 2005, with the exception of 1997. In 1995, 1999, and 2003 it was detected in 3 pilots each year. The average age of these pilots was 56 years (range 34 to 83). Of the narcotic analgesics, propoxyphene and meperidine have the fewest ED mentions from data compiled by DAWN in 2000. ${ }^{14}$ Meperidine and propoxyphene use in pilots during the examined time period is congruent with that compiled from the general population of the United States.

\section{Ketamine}

Ketamine is a central nervous system depressant that produces a rapid-acting dissociative effect. It is an anesthetic that can be injected, snorted, or smoked. It was approved for both human and animal medical use in $1970 .^{3}$ About $90 \%$ of the ketamine sold legally 
today is intended for veterinary use. Large doses lead to reactions similar to those associated with use of phencyclidine (PCP), such as dream-like states and altered perceptions, or hallucinations. ${ }^{3}$ Ketamine was seen in two male pilots: in 1991 in a 29 year-old and in 1994 in a 70 year-old. Ketamine has been marketed in the U. S. for many years; however, it was only recently associated with significant diversion/abuse and was placed in CSA Schedule III in $1999 .^{3}$

\section{DISCUSSION}

From 1990 to 2005, specimens from 5,321 pilots were involved in civil aviation accidents, and these specimens were received by CAMI for toxicological analysis. Of the 5,321 pilots, 5,192 were male (97\%) and 129 were female (3\%). These numbers included both fatal $(5,269 ; 5,141$ males and 128 females) and nonfatal accidents (52; 51 males and 1 female).

\section{Female Pilots}

Of the 129 female pilots involved in aviation accidents, 6 were found positive for one or more of the substances examined in this study. Alprazolam and its active metabolite, alpha-hydroxyalprazolam, were detected in a 36-year-old female pilot in 1994. Diazepam was detected in a 53-year-old in 2005. Pentobarbital was detected in a 37-year-old female pilot in 2001. Hydrocodone was present in a 58-year-old pilot in 2000. Morphine was detected in a 38-year-old female pilot in 2001. Cocaine and its metabolite benzoylecgonine were detected in a 33-year-old female pilot in 1999. The number of female pilots involved in aviation accidents that were received by CAMI for toxicological analysis was small. Due to the lack of data on female pilots testing positive for one of the examined compounds, it is not possible to determine any trends in drug use compared with the general population.

\section{Trends in Drug Use}

Trends in illicit and prescription drug use in pilots involved in civil aviation accidents resemble those seen in emergency departments and community data from major metropolitan areas collected by DAWN and CEWG. Current data collected by DAWN shows that
ED visits involving only illicit drugs accounted for $31 \%$ of the 1.4 million visits related to drug misuse/abuse. ${ }^{12}$ ED visits involving non-medical use of pharmaceuticals accounted for another $27 \%$. Marijuana or its metabolite, THCA, were the most commonly seen illegal compounds detected in pilots involved in civil aviation accidents (Table 1). These compounds were seen approximately twice as often as all other drugs in the present study. This closely resembles the statistics seen in the general population over the same time period.

The average age of the pilots found positive for the compounds discussed in this study was typically older than that seen in EDs or in CEWG communities. However, data from DAWN has reflected an age shift of illicit drug users. The percentage of drug users age 35 and older steadily increased between 1979 (10\%) and 1994 (29\%). Individuals from the heavy drug using cohorts of the 1970s have aged and some continue to use illicit drugs. This has diminished observable reductions in use among the 35 and older age group, resulting in a shift in the age distribution of the population of illicit drug users. ${ }^{12}$

\section{CONCLUSION}

The occurrence of illicit and legal drugs in pilots involved in civil aviation accidents during the examined time period reflected that seen in the non-flying public. There was a slight difference in the average age of the user, with pilots being slightly older on average than other drug users in the U.S. Of 5,321 pilots involved in aviation accidents, there were 467 occurrences of either illicit drugs or commonly abused prescription drugs accounting for $11 \%$ of all pilots that were involved in aviation accidents. As with the general population, the use of marijuana by pilots was far more prevalent than the use of all other illegal and prescription drugs. In fact, marijuana was seen two times as often as the next most used compound. Following marijuana use, the most often used drugs were found to be opiates, benzodiazepines, and cocaine. Drug use in the pilot population was not overly common during the examined time period, as only 1 out of 10 pilots was found positive for one of the drugs included in this study. However, when drugs were detected, the compounds closely followed the trends established in the general population. 


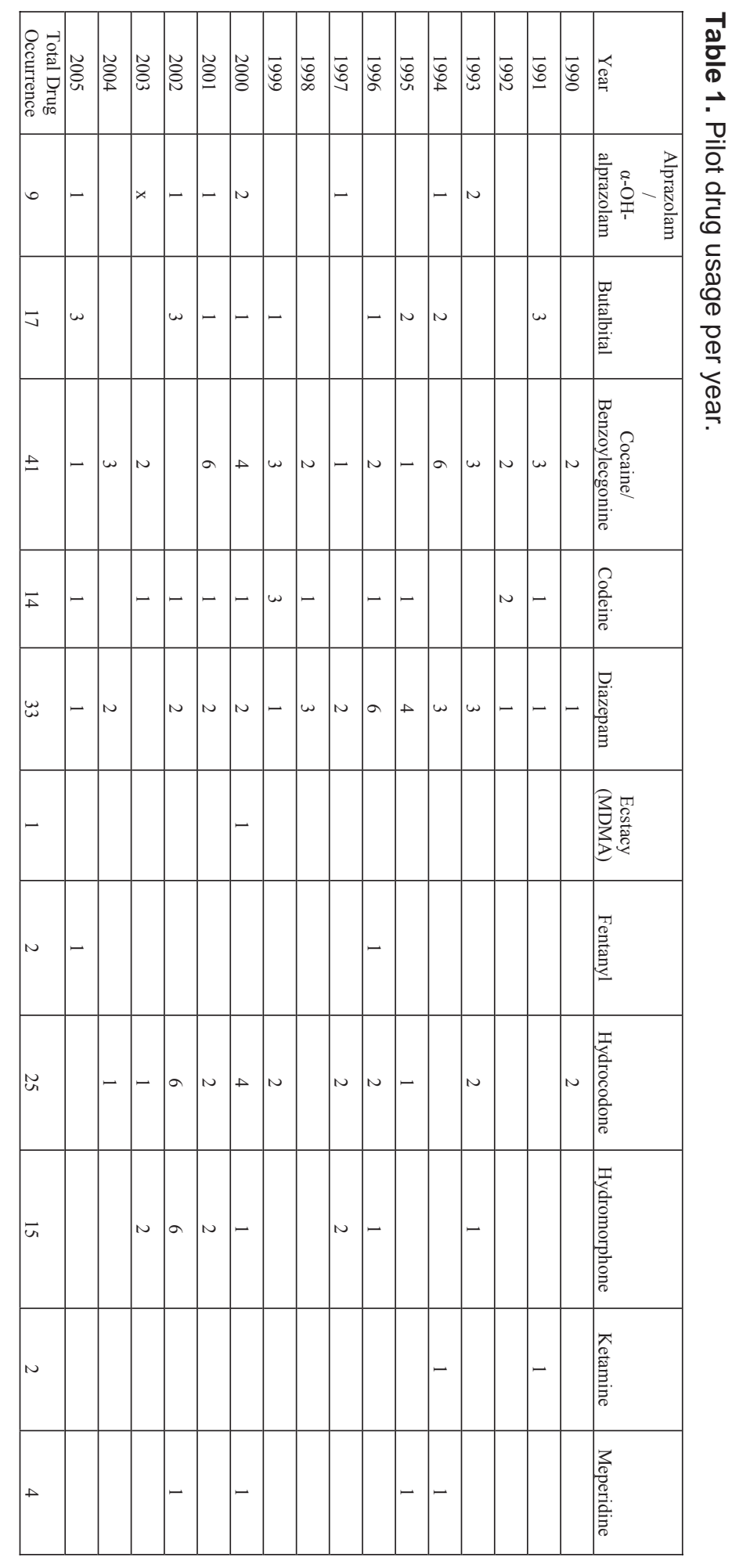




\begin{tabular}{|c|c|c|c|c|c|c|c|c|c|c|c|c|c|c|c|c|}
\hline 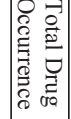 & 嚆 & 蒿 & 莕 & : & & : & : & $\overrightarrow{0}$ & 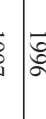 & $\vec{b}$ & $\overrightarrow{0}$ & $\vec{b}$ & 胥 & & $\vec{\circ}$ & 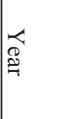 \\
\hline$\widetilde{w}$ & - & n & & -4 & $\omega$ & & $-\mid-$ & . n & - & $-1 N$ & N & - & - & N & & 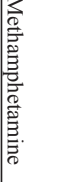 \\
\hline- & & & & & & & & & & & & & - & & & 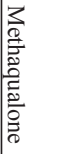 \\
\hline ¿ิ & + & $\omega$ & 6 & n & $\checkmark$ & $\omega \mid \nu$ & $\checkmark+$ & $-1+$ & -+ & $\omega$ & $\omega$ & N & $\omega$ & $\omega$ & & 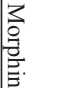 \\
\hline N & & & - & & & & & - & & & & & & & & 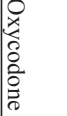 \\
\hline a & & & & N & & & & $\omega$ & & & & & & - & & 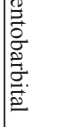 \\
\hline$\sigma$ & & & & & & & - & & & & & - & $N$ & N & & 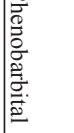 \\
\hline$\infty$ & N & - & $\omega$ & - & N & - $\mid \omega$ & - |- مـ & & - & - $\omega$ & & & & & & 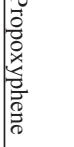 \\
\hline- & & & & & & & & & & & - & & & & & 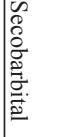 \\
\hline ì & + & N & $\overrightarrow{0}$ & n & $\sigma$ & un un & u & u & 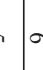 & $\omega$ & $\sigma$ & N & $N$ & N & - & ت्ञ \\
\hline 视 & 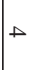 & $\omega$ & $\exists$ & $\checkmark$ & 6 & $\sim \mid \sim$ & $\checkmark a$ & 0 & 0 & 5. & $\mid \vec{N}$ & $\checkmark$ & $\infty$ & 6 & $\checkmark$ & 8불 \\
\hline 志 & 心 & $\vec{v}$ & t & $\breve{\omega}^{\prime}$ & N & $\omega$ & $\vec{\Delta}$ & 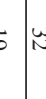 & 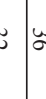 & $\sim$ & $\omega_{\infty}$ & $\tilde{+}$ & $N$ & $\infty$ & I & 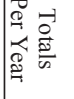 \\
\hline
\end{tabular}




\section{REFERENCES}

1. Aviation Safety Research Act. Aviation Safety Research Act of 1988, Public Law 100-591 [H.R. 4686] 100th U.S. Cong. 2nd Sess., 102 Stat. 3011 (1988).

2. Chaturvedi, A.K., Smith, D.R., Soper, J.W., and Canfield, D.V. Characteristics and Toxicological Processing of Postmortem Pilot Specimens from Fatal Civil Aviation Accidents. Federal Aviation Administration Office of Aerospace Medicine Report No. DOT/FAA/AM-02/14: (2002).

3. DEA Drugs of Abuse. Drugs of Abuse. U.S. Department of Justice. (2005).

4. SAMHSA 1994-2001. Results from the $2001 \mathrm{Na}-$ tional Household Survey on Drug Abuse: Volume II. Technical Appendices and Selected Data Tables (Office of Applied Studies, NHSDA Series H-18) DHHS Publication No. SMA 02-3759: (2002).

5. NIDA Notes: Cocaine. National Institute of Drug Abuse Notes: Facts about Cocaine Abuse and Treatment. 10(5): September/October (1995).

6. NIDA Cocaine Abuse. National Institute on Drug Abuse Research Report Series: Cocaine Abuse and Addiction. NIH Publication No. 99-4342 (1999).

7. SAMHSA ED Trends. Substance Abuse and Mental Health Services Administration, Office of Applied Studies. Emergency Department Trends from the Drug Abuse Warning Network, Final Estimates 1994-2001. DAWN Series D-21: (2002).
8. SAMHSA. Substance Abuse and Mental Health Services Administration. Results from the 2001 National Household Survey on Drug Abuse: Volume II. Technical Appendices and Selected Data Tables. NHSDA Series H-18, DHHS Publication No. SMA 02-3759: (2002).

9. NIDA Research Report MDMA. National Institute on Drug Abuse Research Report - MDMA Abuse (Ecstasy) NIH Publication No. 05-4728: (2005).

10. Drug Abuse Warning Network. The DAWN Report: Club Drugs, 2002 Update. (2004).

11. SAMHSA National Estimates. Drug Abuse Warning Network, 2005: National Estimates of DrugRelated Emergency Department Visits. DAWN Series D-29: (2005).

12. Community Epidemiology Work Group, Epidemiologic Trends in Drug Abuse. Advance Report and Highlights/Executive Summary: Abuse of Stimulants and Other Drugs. NIH Publication No. 05-5280: (2005).

13. NIDA Prescription Drugs. National Institute on Drug Abuse Research Report - Prescription Drugs: Abuse and Addiction. NIH Publication No. 014881: (2001).

14. NIDA Epidemiologic Trends. Epidemiologic Trends in Drug Abuse Volume I 2002. National Institute on Drug Abuse, National Institutes of Health, Department of Health and Human Services. NIH Publication No. 03-5365: (2002).

15. Office of Diversion Control. Office of Diversion Control. U.S. Department of Justice, Drug Enforcement Administration. Drug Chemicals of Concern. DEA/OD/ODE: (2006). 